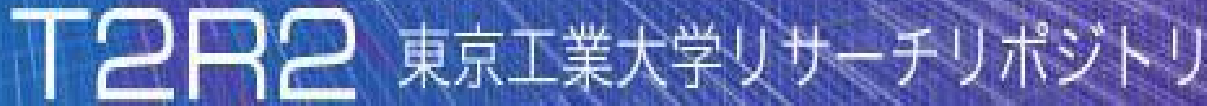

\section{Tokyo Tech Research Repository}

\section{論文 /著書情報 \\ Article /Book Information}

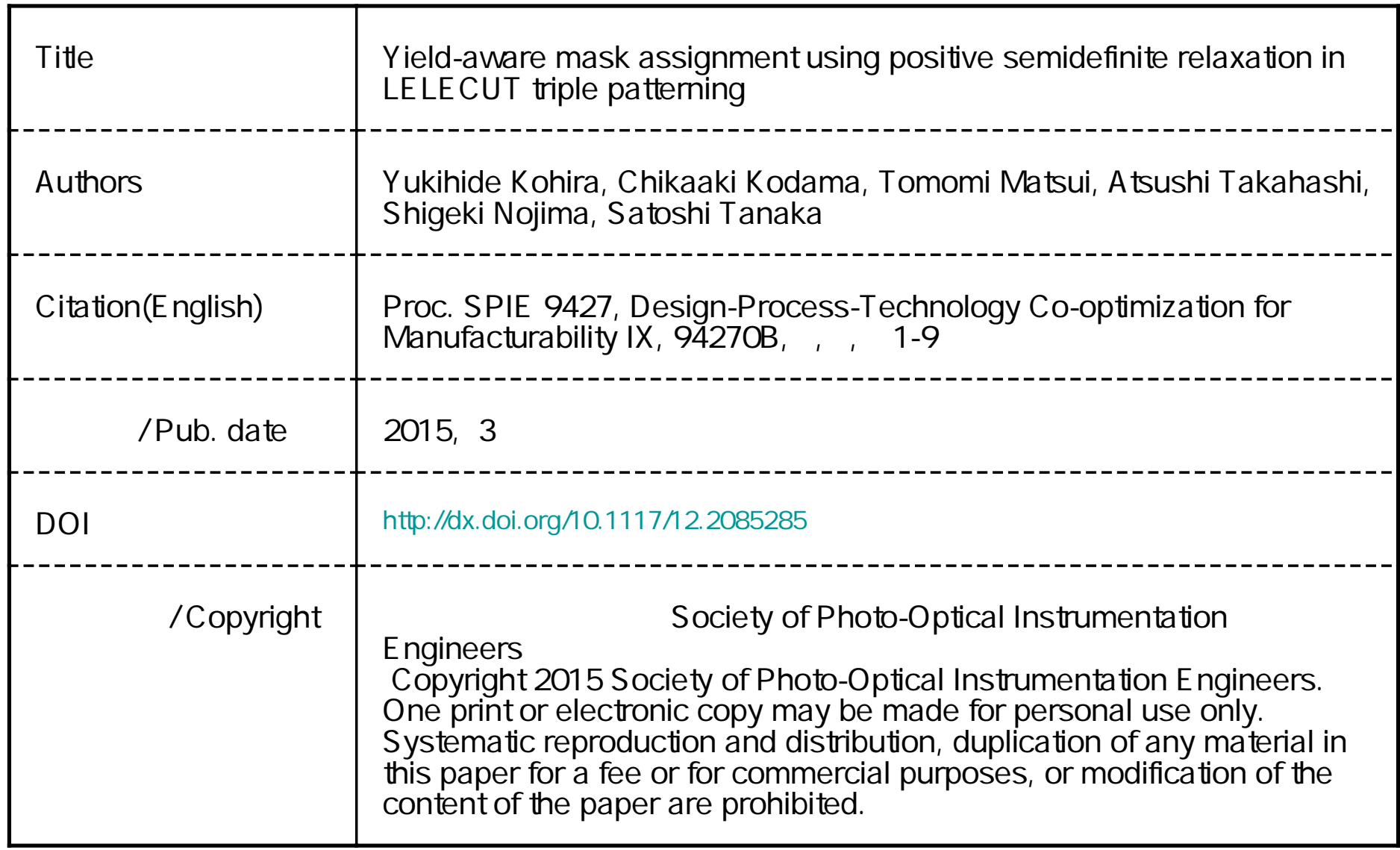




\title{
Yield-aware Mask Assignment using Positive Semidefinite Relaxation in LELECUT Triple Patterning
}

\author{
Yukihide Kohira $^{a}$, Chikaaki Kodama ${ }^{b}$, Tomomi Matsui ${ }^{c}$, \\ Atsushi Takahashi ${ }^{c}$, Shigeki Nojima ${ }^{b}$ and Satoshi Tanaka ${ }^{b}$ \\ ${ }^{a}$ The University of Aizu, Aizu-Wakamatsu, Japan \\ ${ }^{b}$ Toshiba Corporation, Yokohama, Japan \\ ${ }^{c}$ Tokyo Institute of Technology, Tokyo, Japan
}

\begin{abstract}
LELECUT type triple patterning lithography is one of the most promising techniques in the next generation lithography. To prevent yield loss caused by overlay error, LELECUT mask assignment which is tolerant to overlay error is desired. In this paper, we propose a method that obtains an LELECUT assignment which is tolerant to overlay error. The proposed method uses positive semidefinite relaxation and randomized rounding technique. In our method, the cost function that takes the length of boundary of features determined by the cut mask into account is introduced.
\end{abstract}

Keywords: Triple Patterning, LELECUT, Design for Manufacturability, Positive Semidefinite Relaxation

\section{INTRODUCTION}

Multiple patterning technique enables us to fabricate small features without using advanced technologies such as extreme ultra violet (EUV) lithography. Triple patterning lithography (TPL) is one of the most promising techniques in $14 \mathrm{~nm}$ logic node and beyond. In order to realize a target pattern, various types of techniques including design for manufacturability such as LELE type double patterning lithograph,${ }^{1-7}$ LELELE type TPL ${ }^{8-18}$ LELECUT type TPL, ${ }^{19,20}$ and side wall process, ${ }^{21}$ are used in addition to a basic litho-etch process with optimized mask. These techniques are summarized in. ${ }^{22,23}$

Sidewall process ${ }^{21}$ forms a wall feature with unique width so that it surrounds the prefabricated polygon. The sidewall process which is used in self-aligned double patterning enables us to fabricate finer pattern pitch by combining a slimming process, but the variety of target patterns that can be fabricated is limited.

Two types of TPL technologies are often discussed in literature. In LELELE, litho-etch process is repeated three times. However, it is difficult to achieve high yield due to native conflict and overlay problems. In LELECUT, the third mask called cut (or trim) process removes a part of a fabricated pattern. It is used to improve the quality of fabricated patterns as well as to enhance the flexibility of layout. However, it has overlay problems and lithographical limitations. In order to prevent yield loss caused by overlay error as much as possible, LELECUT mask assignment which is tolerant to overlay error is desired.

To our best knowledge, two LELECUT mask assignment methods have been proposed. In, ${ }^{19}$ LELECUT mask assignment problem is formulated as an integer linear programming problem. Although it minimizes the weighted summation of the number of conflicts and stitches, the effect of cuts on layout quality is not taken into account. In,$^{20}$ LELECUT mask assignment problem is solved by positive semidefinite relaxation. Although it minimizes the weighted summation of the number of conflicts, stitches, and polygons in the cut mask, the yield of obtained layout is also not discussed. Fig. 1 shows mask assignments in LELECUT. A target pattern is shown in Fig. 1 (a). The layouts obtained by two LELECUT mask assignments which are represented by blue, magenta, and cut masks without overlay error are shown in Fig. 1 (b) and Fig. 1 (d). These mask assignments have no

Further author information: (Send correspondence to Yukihide Kohira, Chikaaki Kodama and Atsushi Takahashi) Yukihide Kohira: E-mail:kohira@u-aizu.ac.jp, Telephone: +81-242-37-2536

Chikaaki Kodama: E-mail:chikaaki1.kodama@toshiba.co.jp, Telephone: +81-45-890-2818

Atsushi Takahashi: E-mail:atsushi@eda.ce.titech.ac.jp, Telephone: +81-3-5734-2665

Design-Process-Technology Co-optimization for Manufacturability IX, edited by John L. Sturtevant, Luigi Capodieci, Proc. of SPIE Vol. 9427, 94270B · @ 2015 SPIE · CCC code: 0277-786X/15/\$18 · doi: 10.1117/12.2085285 


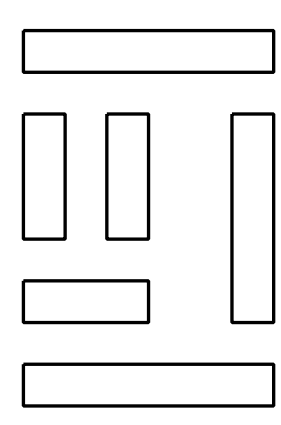

(a) A target pattern.

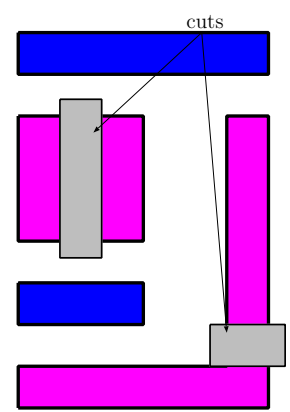

(b) A mask assignment.

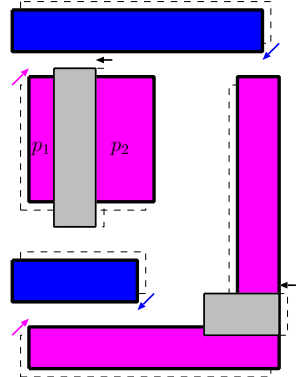

(c) Mask assignment shown in (b) with misalignment.

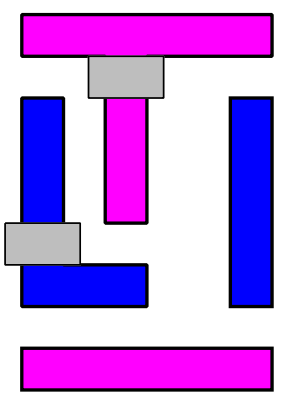

(d) Another mask assignment.

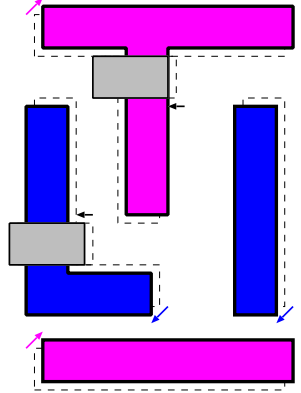

(e) Mask assignment shown in (d) with misalignment.

Figure 1. Mask assignments in LELECUT. Mask assignment shown in (c) has higher yield than that shown in (d).

conflicts, no stitches, and the number of polygons in the cut mask is same. The layouts of them with overlay error in which blue, magenta, and cut masks move to the lower left, the upper right, and the left, respectively, are shown in Fig. 1 (c) and Fig. 1 (e), respectively. The former is expected to have lower yield than the latter since a longer dimension of features such as $p_{1}$ and $p_{2}$ is determined by the cut mask and is affected directly by the overlay error. The length of a boundary of a feature that is determined by the cut mask should be small enough to prevent the yield loss caused by overlay error.

In this paper, we propose a method that obtains an LELECUT assignment which is tolerant to overlay error. The proposed method is an enhancement of the method proposed $i^{20}$ and uses positive semidefinite relaxation and randomized rounding technique. In our method, the cost function that takes the length of boundary of features determined by the cut mask into account is introduced to obtain an overlay tolerant LELECUT assignment.

\section{PRELIMINARIES}

\subsection{Problem Definition}

Let $P=\left\{p_{1}, p_{2}, \ldots, p_{n}\right\}$ be the set of polygons in a target pattern. A polygon may represent a polygon decomposed by given stitch candidates. A stitch edge is defined between two polygons if and only if two polygons are decomposed by a stitch candidate. A polygon conflict edge is defined between two polygons if and only if two polygons are too close to assign the same mask. A cut candidate is defined between two polygons connected by a polygon conflict edge if and only if they can be cut by the cut mask when they are assigned to the same mask. A cut candidate $c$ has a cost $l(c)$ which is defined by the length of boundary between polygons in the target pattern and the cut candidate. A cut candidate may not be independent of other cut candidates. A cut conflict edge is defined between two cut candidates if and only if they cannot be used simultaneously. Note that even if the distance between two cut candidates is not long enough, they can be used simultaneously if they can be merged into one. A cut conflict edge is not defined between two cut candidates if the distance between them is long enough or if they can be merged into one without affecting the critical dimension of pattern. Let $S, C_{P}, T$, and $C_{T}$ be the set of stitch edges, the set of polygon conflict edges, the set of cut candidates, and the set of cut conflict edges, respectively. Note that both the set of stitch edges $S$ and the set of polygon conflict edges $C_{P}$ are families of unordered pairs of polygons in $P$, the set of cut candidates $T$ is a sub-set of the set of polygon conflict edges $C_{P}$, and the set of cut conflict edges $C_{T}$ is a family of unordered pairs of cut candidates in $T$.

Fig. 2 shows an example of problem. In this example, $P=\left\{p_{1}, p_{2}, p_{3}, p_{4}\right\}, S=\left\{\left\{p_{3}, p_{4}\right\}\right\}, C_{P}=T=$ $\left\{c_{1}, c_{2}, c_{3}, c_{4}, c_{5}\right\}$, where $c_{1}=\left\{p_{1}, p_{3}\right\}, c_{2}=\left\{p_{1}, p_{4}\right\}, c_{3}=\left\{p_{1}, p_{2}\right\}, c_{4}=\left\{p_{2}, p_{3}\right\}$, and $c_{5}=\left\{p_{2}, p_{4}\right\}$ with costs $l\left(c_{1}\right)=2, l\left(c_{2}\right)=2, l\left(c_{3}\right)=1, l\left(c_{4}\right)=2$, and $l\left(c_{5}\right)=2$. The set of cut conflict edges is given by $C_{T}=\left\{\left\{c_{1}, c_{2}\right\},\left\{c_{1}, c_{3}\right\},\left\{c_{2}, c_{3}\right\},\left\{c_{3}, c_{4}\right\},\left\{c_{3}, c_{5}\right\},\left\{c_{4}, c_{5}\right\}\right\}$.

In this paper, a polygon is assigned to one of two masks except the cut mask. The problem of finding an assignment of polygons, and/or two-coloring, is essentially equivalent to a maximum cut problem. We employ a 


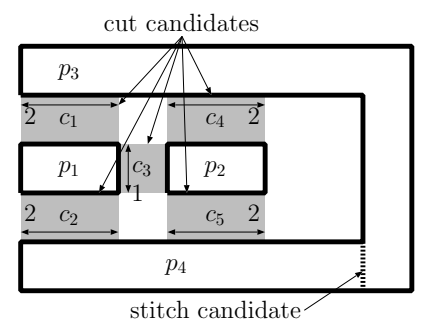

Figure 2. Polygons with stitch and cut candidates.

$\{-1,1\}$ formulation represented by function $x: P \rightarrow\{-1,1\}$, which is introduced by Goemans and Williamson $i^{24}$ for a max cut problem. This formulation naturally yields a positive semidefinite relaxation appearing in a later section. For two-coloring $x$, the set of polygon conflict edges that connect the same color polygons is represented by

$$
C_{P}(x) \stackrel{\text { def. }}{=}\left\{\{p, q\} \in C_{P} \mid x(p)=x(q)\right\} .
$$

Similarly, the set of stitch candidates that connect the different color polygons is represented by

$$
S(x) \stackrel{\text { def. }}{=}\{\{p, q\} \in S \mid x(p) \neq x(q)\} .
$$

The set of feasible cuts for two-coloring $x$ is a subset of $C_{P}(x) \cap T$ and an independent set of cut graph $\left(T, C_{T}\right)$. The problem we consider is given as follows:

\section{P1: minimize}

$$
\begin{aligned}
& \alpha_{1}\left|C_{P}(x) \backslash T^{\prime}\right|+\alpha_{2}|S(x)|+\alpha_{3} \sum_{c \in T^{\prime}} l(c) \\
= & \alpha_{1}\left|C_{P}(x)\right|+\alpha_{2}|S(x)|-\alpha_{1} \sum_{c \in T^{\prime}} 1+\alpha_{3} \sum_{c \in T^{\prime}} l(c) \\
= & \alpha_{1}\left|C_{P}(x)\right|+\alpha_{2}|S(x)|+\sum_{c \in T^{\prime}}\left(\alpha_{3} l(c)-\alpha_{1}\right)
\end{aligned}
$$

subject to

- $x(p) \in\{-1,1\} \quad(\forall p \in P)$,

- $T^{\prime} \subseteq C_{P}(x) \cap T$,

- $T^{\prime}$ is an independent set of cut graph $\left(T, C_{T}\right)$.

In this formulation, the weighted sum of the number of unresolved conflict edges, the number of caused stitches, and the total cost of used cut candidates is minimized. In the following, we assume that $\alpha_{1} \geq \alpha_{2} \geq 0$ and $\alpha_{1} \geq \alpha_{3} l(c) \geq 0$. According to this assumption, the total cost of used cut candidates is minimized under the condition that the number of conflict edges that connect the same polygons is minimized and the number of used cut candidates is maximized.

Fig. 3 shows examples of mask assignments. The mask assignment shown in Fig. 3 (a) has two cuts with total cost 4 and one stitch. On the other hand, that shown in Fig. 3 (b) has one cut with total cost 1. Obviously, the mask assignment shown in Fig. 3 (b) is better than that shown in Fig. 3 (a).

\subsection{Maximum Independent Set with Minimum Total Cost Problem}

For a given two-coloring $x: P \rightarrow\{-1,1\}$, the problem $\mathrm{P} 1$ is equivalent to a maximum independent set with minimum total cost problem MISMTCP1 since we assume $\alpha_{1} \geq \alpha_{3} l(c)$. 


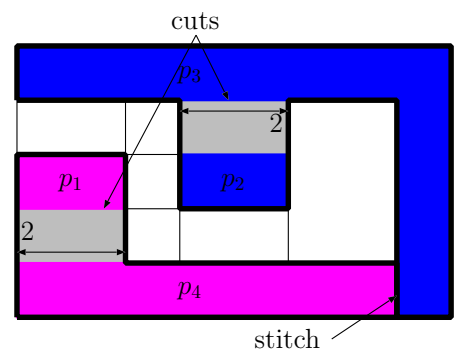

(a) Two cuts with total cost 4 and one stitch.

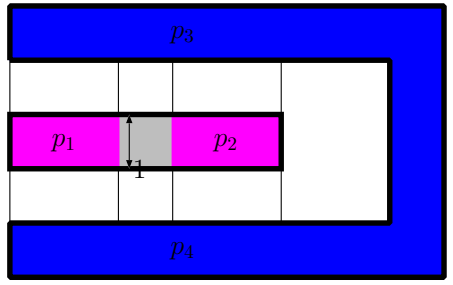

(b) One cut with total cost 1 .

Figure 3. Mask assignments for layout in Fig. 2.

Step 1: Formulate a positive semidefinite relaxation SDP-L.

Step 2: Solve SDP-L by SDP solver.

Step 3: Obtain a mask assignment by randomized rounding algorithm with iterative improvement.

Figure 4. Outline of the proposed method.

\section{MISMTCP1: maximize}

$$
\sum_{c \in T^{\prime}}(1-\alpha l(c))
$$

subject to

- $\alpha l(c)<1$

- $T^{\prime} \subseteq C_{P}(x) \cap T$,

- $T^{\prime}$ is an independent set of cut graph $\left(T, C_{T}\right)$.

A maximum independent set with minimum total cost problem is known to be NP-hard. In this paper, the following 0-1 integer linear programming MISMTCP2 is formulated by introducing $0-1$ variable $y(c)$.

\section{MISMTCP2: maximize}

$$
\sum_{c \in T} y(c) \cdot(1-\alpha l(c))
$$

subject to

$$
\begin{aligned}
y(c) \in\{0,1\} & (\forall c \in T), \\
y(c)+y\left(c^{\prime}\right) \leq 1 & \left(\forall\left\{c, c^{\prime}\right\} \in C_{T}\right), \\
y(c)=0 & \left(\forall c \in T \backslash C_{P}(x)\right) .
\end{aligned}
$$

\section{POSITIVE SEMIDEFINITE RELAXATION}

\subsection{Outline of Proposed Method}

The outline of the proposed method is shown in Fig. 4. The proposed method is based on the method proposed in. $^{20}$ In the proposed method, a positive semidefinite relaxation of P1 called SDP-L is formulated and a mask assignment is obtained from an optimum solution of the relaxation by randomized rounding technique with iterative improvement. It is well-known that a positive semidefinite programming problem can be solved by interior point methods in polynomial time. 


\subsection{Our Semidefinite Relaxation}

In this subsection, we introduce a positive semidefinite relaxation of P1. Our relaxation is an enhancement of the formulation proposed by Goemans and Williamson ${ }^{24}$ for a max cut problem to handle a maximum independent set with minimum total cost.

First, we represent the objective function of $\mathrm{P} 1$ as a linear function. An arbitrary two-coloring $x: P \rightarrow$ $\{-1,1\}$, satisfies: $x(p)=x(q) \leftrightarrow x(p) x(q)=1$ and $x(p) \neq x(q) \leftrightarrow x(p) x(q)=-1$. By using these properties, $\left|C_{P}(x)\right|$ and $|S(x)|$ are represented in terms of $x$ as follows:

$$
\begin{aligned}
\left|C_{P}(x)\right| & =\sum_{\{p, q\} \in C_{P}}\left(\frac{x(p) x(q)}{2}+\frac{1}{2}\right) \\
|S(x)| & =\sum_{\{p, q\} \in S}\left(-\frac{x(p) x(q)}{2}+\frac{1}{2}\right) .
\end{aligned}
$$

Let $X$ be the $n \times n$ matrix whose $(p, q)$-th element is $X_{p q}=x(p) x(q)(\forall p, \forall q \in P)$. Let $C$ and $S$ be matrixes that represent polygon conflicts and stitch candidates, respectively. That is, $C$ and $S$ are the $n \times n$ symmetric matrixes, and

$$
C_{p q}=\left\{\begin{array}{cc}
\frac{1}{4} & \left(\{p, q\} \in C_{P}\right), \\
0 & \left(\{p, q\} \notin C_{P}\right),
\end{array} \quad S_{p q}=\left\{\begin{array}{cc}
\frac{1}{4} & (\{u, v\} \in S), \\
0 & (\{u, v\} \notin S),
\end{array}\right.\right.
$$

respectively. Then, we have

$$
\begin{aligned}
& \left|C_{P}(x)\right|=\sum_{\{p, q\} \in C_{P}}\left(\frac{x(p) x(q)}{2}+\frac{1}{2}\right)=C \bullet X+\text { const, } \\
& |S(x)|=\sum_{\{p, q\} \in S}\left(-\frac{x(p) x(q)}{2}+\frac{1}{2}\right)=-S \bullet X+\text { const, }
\end{aligned}
$$

where $M \bullet M^{\prime}$ is defined as $\sum_{i} \sum_{j} M_{i j} M_{i j}^{\prime}$ for square matrixes $M$ and $M^{\prime}$ of the same size. Let $y(c)$ be a $0-1$ variable for a cut candidate $c$. $c$ is assigned to the cut mask, if and only if $y(c)=1$. Then, we have

$$
\sum_{c \in T^{\prime}}\left(\alpha_{3} l(c)-\alpha_{1}\right)=\sum_{c \in T} y(c) \cdot\left(\alpha_{3} l(c)-\alpha_{1}\right) .
$$

Therefore, the objective function of $\mathrm{P} 1$ is represented as

$$
\alpha_{1}(C \bullet X)-\alpha_{2}(S \bullet X)+\sum_{c \in T} y(c) \cdot\left(\alpha_{3} l(c)-\alpha_{1}\right)+\text { const. }
$$

Note that $C$ and $S$ are constant matrixes.

Next, constraints of P1 are represented as linear functions in terms of $X$ and $y$. Constraint (3) in MISMTCP2 is represented as

$$
0 \leq y(c) \leq \begin{cases}1 & \left(\text { if } c \in T \cap C_{P}(x)\right) \\ 0 & \left(\text { if } c \in T \backslash C_{P}(x)\right)\end{cases}
$$

Then, constraint (3) is represented by using two-coloring $x$ as follows:

$$
0 \leq y(c) \leq \frac{x(p) x(q)}{2}+\frac{1}{2} \quad(\forall c=\{p, q\} \in T) .
$$

The representations of other constraints are straightforward, and P1 is represented as follows: 


\section{P2: minimize}

$$
\alpha_{1}(C \bullet X)-\alpha_{2}(S \bullet X)+\sum_{c \in T} y(c) \cdot\left(\alpha_{3} l(c)-\alpha_{1}\right)
$$

subject to

$$
\begin{aligned}
X_{p q}=x(p) x(q) & \left(\forall(p, q) \in P^{2}\right), \\
x(p) \in\{-1,1\} & (\forall p \in P), \\
y(c) \in\{0,1\} & (\forall c \in T), \\
y(c)+y\left(c^{\prime}\right) \leq 1 & \left(\forall\left\{c, c^{\prime}\right\} \in C_{T}\right), \\
0 \leq y(c) \leq \frac{x(p) x(q)}{2}+\frac{1}{2} & (\forall c=\{p, q\} \in T) .
\end{aligned}
$$

The objective function of P2 is obtained from Eq. (4) by removing constant term. Note that $X_{p p}=1$ for all $p \in P$.

Since $X$ in $\mathrm{P} 2$ is a positive semidefinite symmetric matrix, the problem $\mathrm{P} 2$ has a positive semidefinite programming relaxation as follows. Let $\mathcal{S}_{+}^{n}$ be the set of $n \times n$ positive semidefinite symmetric matrix. A positive semidefinite relaxation problem SDP-L is obtained from P2 by restricting $X$ within $\mathcal{S}_{+}^{n}$ and ignoring 0-1 constraints for $y$, instead of constraints (5), (6), and (7).

\section{SDP-L: minimize}

$$
\alpha_{1}(C \bullet X)-\alpha_{2}(S \bullet X)+\sum_{c \in T} y(c) \cdot\left(\alpha_{3} l(c)-\alpha_{1}\right)
$$

subject to

$$
\begin{aligned}
X_{p p}=1 & (\forall p \in P), \\
y(c)+y\left(c^{\prime}\right) \leq 1 & \left(\forall\left\{c, c^{\prime}\right\} \in C_{T}\right), \\
0 \leq y(c) \leq \frac{1}{2} X_{p q}+\frac{1}{2} & (\forall c=\{p, q\} \in T), \\
X \in \mathcal{S}_{+}^{n} . &
\end{aligned}
$$

SDP-L is a positive semidefinite programming problem and can be solved by interior point methods in polynomial time.

\subsection{Randomized Rounding for LELECUT}

In this subsection, we propose a randomized rounding technique based on the hyper-plane separation technique proposed by Goemans and Wiiliamson, ${ }^{24}$ which gives 0.878 approximation algorithm for a max cut problem. The randomized rounding technique is based on the method proposed in, ${ }^{20}$ and the iterative improvement is applied as post-processing.

For any positive semidefinite symmetric matrix $X \in \mathcal{S}_{+}^{n}$, there exists a matrix $Z$ satisfying $X=Z^{\top} Z$. This decomposition is called Cholesky decomposition.

We solve problem SDP-L by a SDP solver and obtain an optimal solution $(\widetilde{X}, \widetilde{y})$ for SDP-L. Let $\widetilde{Z}^{\top} \widetilde{Z}$ be the Cholesky decomposition of $\widetilde{X}$ and $d$ be the number of rows of $\widetilde{Z}$. Here we note that columns of $\widetilde{Z}$ are indexed by polygons in $P$ and the length of every column vector is equal to 1 . For each polygon $p \in P$, vector $\widetilde{\boldsymbol{z}}(p) \in \mathbb{R}^{d}$ denotes the corresponding column vector of $\widetilde{Z}$. Algorithm RR shown in Fig. 5 outputs a two coloring $\widetilde{x}: P \rightarrow\{-1,1\}$ and the set $\widetilde{T}$ of cuts. The mask assignment is modified by the greedy iterative improvement so that better solutions are obtained. The quality of the obtained mask assignment depends on the generated random unit vector and the runtime of Algorithm RR is very small. Therefore, Algorithm RR is repeated appropriate times and the best mask assignment is output. During the repetition, we might choose a comfortable mask assignment as well. 


\begin{abstract}
Algorithm RR
Step 1: Generate a random unit vector $\boldsymbol{u} \in \mathbb{R}^{d}$ (satisfying $\|\boldsymbol{u}\|=1$ ).

Step 2: For each polygon $p \in P$, set $\widetilde{x}(p)= \begin{cases}1 & \left(\text { if } \boldsymbol{u}^{\top} \widetilde{\boldsymbol{z}}(p)>0\right), \\ -1 & \text { (otherwise) }\end{cases}$

Step 3: Construct a subgraph $\widetilde{G}$ of cut graph $\left(T, C_{T}\right)$ induced by vertex subset $C_{P}(\widetilde{x}) \cap T$. Find a maximal independent set $\widetilde{S}$ of $\widetilde{G}$ by employing a heuristic algorithm for maximum independent set with minimum total cost problem.

Step 4: Apply the greedy iterative improvement in which mask assignment of a polygon is changed and a heuristic algorithm for maximum independent set with minimum total cost problem is applied until the solution is not improved.
\end{abstract}

Figure 5. Randomized Rounding Algorithm.

Table 1. Experimental results. The obtained mask assignments have no polygon conflicts and cut conflicts in ILP-\#, ILP-L, SDP-\#, and SDP-L Imp. The units of cost and time are [nm] and [s], respectively.

\begin{tabular}{|c|c|c|c|c|c|c|c|c|c|c|c|c|c|c|c|c|c|c|c|c|c|c|}
\hline \multirow[b]{2}{*}{ circuit } & \multirow[b]{2}{*}{$|P|$} & \multirow[b]{2}{*}{ \# Seg } & \multirow[b]{2}{*}{$\left|C_{P}\right|$} & \multicolumn{2}{|c|}{ \# comp } & \multirow[b]{2}{*}{$|T|$} & \multicolumn{3}{|c|}{ ILP-\# } & \multicolumn{3}{|c|}{ ILP-L } & \multicolumn{3}{|c|}{ SDP-\# } & \multicolumn{4}{|c|}{ SDP-L } & \multicolumn{3}{|c|}{ SDP-L Imp } \\
\hline & & & & total & target & & $\left|T^{\prime}\right|$ & cost & time & $\left|T^{\prime}\right|$ & cost & time & $\left|T^{\prime}\right|$ & cost & time & $\left|T^{\prime}\right|$ & $\left|C_{P}(x)\right|$ & cost & time & $\left|T^{\prime}\right|$ & cost & time \\
\hline c432 & 850 & 4918 & 540 & 414 & 1 & 12 & 1 & 40 & 0.01 & 1 & 40 & 0.01 & 1 & 40 & 0 & 1 & 0 & 40 & 0 & 1 & 40 & 0 \\
\hline c499 & 1491 & 9518 & 1489 & 502 & 50 & 278 & 58 & 2720 & 0.42 & 58 & 2720 & 0.45 & 58 & 6030 & 0.12 & 62 & 0 & 2940 & 0.12 & 58 & 2720 & 0.15 \\
\hline c 880 & 1872 & 10666 & 1422 & 717 & 168 & 934 & 172 & 11720 & 2.46 & 198 & 10390 & 6.25 & 172 & 20590 & 0.44 & 202 & 2 & 11105 & 0.35 & 198 & 10390 & 0.51 \\
\hline c1355 & 2656 & 15246 & 1514 & 1328 & 76 & 514 & 90 & 9760 & 1.10 & 122 & 8320 & 6.10 & 90 & 13230 & 0.27 & 130 & 2 & 9635 & 0.17 & 122 & 8320 & 0.37 \\
\hline c1908 & 4191 & 24370 & 3141 & 1733 & 182 & 1462 & 211 & 37700 & 5.77 & 373 & 30410 & 24.34 & 211 & 41140 & 0.73 & 411 & 13 & 36600 & 0.43 & 373 & 30410 & 1.37 \\
\hline c 2670 & 6371 & 37564 & 5802 & 2056 & 585 & 4298 & 686 & 76950 & 10.52 & 958 & 64710 & 38.98 & 686 & 101975 & 2.32 & 1069 & 66 & 84540 & 1.39 & 952 & 65775 & 5.26 \\
\hline c3540 & 8188 & 47244 & 6897 & 2896 & 775 & 4794 & 821 & 73560 & 11.06 & 1044 & 63445 & 29.61 & 821 & 111330 & 2.24 & 1090 & 11 & 72630 & 1.47 & 1044 & 63445 & 3.02 \\
\hline c5315 & 11498 & 68476 & 10097 & 3926 & 1193 & 7552 & 1259 & 108290 & 17.52 & 1572 & 93965 & 53.23 & 1259 & 162510 & 3.59 & 1647 & 13 & 106280 & 2.33 & 1572 & 93965 & 4.11 \\
\hline c6288 & 11605 & 64762 & 5602 & 6259 & 256 & 1282 & 256 & 10320 & 2.39 & 256 & 10240 & 2.33 & 256 & 29840 & 0.66 & 256 & 0 & 10240 & 0.45 & 256 & 10240 & 0.66 \\
\hline ave. & & & & & & & 0.82 & 1.12 & 0.50 & (1) & (1) & (1) & 0.82 & 1.77 & 0.09 & 1.05 & & 1.12 & 0.07 & 1.00 & 1.00 & 0.12 \\
\hline
\end{tabular}

$|P| \quad$ the number of polygons

\# Seg the number of line segments

$\left|C_{P}\right| \quad$ the number of polygon conflict edges

total the number of components in the conflict graph $\left(P, C_{P}\right)$

target the number of components in the conflict graph $\left(P, C_{P}\right)$ in which cuts must be inserted

$|T|$ the number of cut candidates in the target components

$\left|T^{\prime}\right| \quad$ the number of inserted cuts

$\left|C_{P}(x)\right|$ the number of conflicts in the obtained mask assignment

cost the total cost of cuts in the obtained mask assignment

time computational time

ave. the average normalized by ILP-L

\title{
4. EXPERIMENTS
}

Our proposed mask assignment method is implemented by using a SDP solver and $\mathrm{C}++$ language. We compare the following five methods. ILP-\# and ILP-L are ILP formulations based on ${ }^{19}$ which minimizes the number of cuts and minimizes the total cost of cuts, respectively. SDP-\# is the positive semidefinite relaxation proposed in $^{20}$ which minimizes the number of cuts. SDP-L Imp and SDP-L are the proposed methods. SDP-L Imp applies the greedy iterative improvement as post-processing and SDP-L does not. The methods are executed on a Linux machine with 12 GB memory by using Intel core i7-3770 of $3.40 \mathrm{GHz}$. In our implementation, SDP problems are solved by SDPA 7.3.8 ${ }^{25}$ which is a free tool. On the other hand, ILP problems are solved by CPLEX 12.6.1 ${ }^{26}$ which is one of the most famous commercial ILP solvers. In these methods, a speedup technique in which the conflict graph $\left(P, S \cap C_{P}\right)$ is decomposed into connected components is adopted. This speedup technique is discussed in many previous studies. ${ }^{1-3,5,6,19,20}$ In this experiment, we do not prepare stitch candidates to focus on observing the total cost, the number of cuts, and the number of conflicts. The parameters in objective functions are set to $\alpha_{1}=10^{6}$ and $\alpha_{3}=1 . \alpha_{1}=10^{6}$ is much larger than cut costs. Algorithm RR is applied 100 times in SDP-\#, SDP-L, and SDP-L Imp.

ISCAS benchmarks which were used in $^{3,5}$ are used. The benchmarks are reproduced from the information given by authors of ${ }^{3,5}$ and from figures in, ${ }^{3}$ though we could not obtain the same data. We followed the 
parameters as in, ${ }^{3,5}$ where the minimum polygon space in a mask is $54 \mathrm{~nm}$. If stitches are allowed to be inserted, the mask assignment without cuts is obtained. Therefore, we also do not insert stitches in this experiment. Table 1 shows the results. Note that the mask assignments obtained by all methods except SDP-L have no polygon conflicts and cut conflicts. Since the minimization of the total cost is added into the objective function of ILP-L, the total cost obtained by ILP-L is optimum. Similarly, since the minimization of cuts is added into the objective function of ILP-\#, the number of cuts obtained by ILP-\# is optimum. Although ILP-\# and SDP-\# obtain mask assignments with the minimum number of cuts, the total cost of the mask assignment obtained by them is larger than that by ILP-L since the minimization of cuts does not corresponds to that of the total cost. Although SDP-L is fast, it obtains mask assignments with conflicts. The total cost of the mask assignment obtained by SDP-L Imp is the same as that by ILP-L in nine circuits of ten circuits. Moreover, SDP-L Imp is much faster than ILP-L. Consequently, SDP-L Imp obtains optimum solutions in the shortest computational time in almost all circuits.

\section{CONCLUSIONS}

In this paper, we propose a fast LELECUT mask assignment method to be tolerant to overlay error. The proposed method applies a positive semidefinite relaxation. The experimental results show the efficiency and the validity of the proposed method. We will take the mask density balance, stitch direction, and etc. into account to improve the quality of the mask assignment in our future works.

\section{ACKNOWLEDGMENTS}

This work was supported by JSPS KAKENHI Grant-in-Aid for Scientific Research (B) 25280013.

\section{REFERENCES}

[1] Kahng, A., Park, C.-H., Xu, X., and Yao, H., "Layout decomposition for double patterning lithography," in [Proc. ICCAD], 465-472 (2008).

[2] Yuan, K., Yang, J.-S., and Pan, D., "Double patterning layout decomposition for simultaneous conflict and stitch minimization," in [Proc. ISPD], 107-114 (2009).

[3] Yang, J.-S., Lu, K., Cho, M., Yuan, K., and Pan, D. Z., "A new graph-theoretic, multi-objective layout decomposition framework for double patterning lithography," in [Proc. ASP-DAC], 637-644 (2010).

[4] Chen, S.-Y. and Chang, Y.-W., "Native-conflict-aware wire perturbation for double patterning technology," in [Proc. ICCAD], 556-561 (2010).

[5] Tang, X. and Cho, M., "Optimal layout decomposition for double patterning technology," in [Proc. ICCAD], 9-13 (2011).

[6] Kohira, Y., Yokoyama, Y., Kodama, C., Takahashi, A., Nojima, S., and Tanaka, S., "Yield-aware decomposition for LELE double patterning," in [Proc. SPIE], 9053, 90530T (2014).

[7] Yokoyama, Y., Sakanushi, K., Kohira, Y., Takahashi, A., Kodama, C., Tanaka, S., and Nojima, S., "Localization concept of re-decomposition area to fix hotspots for LELE process," in [Proc. SPIE], 9053, 90530V (2014).

[8] Yu, B., Yuan, K., Zhang, B., Ding, D., and Pan, D. Z., "Layout decomposition for triple patterning lithography," in [Proc. ICCAD], 1-8 (2011).

[9] Fang, S.-Y., Chang, Y.-W., and Chen, W.-Y., "A novel layout decomposition algorithm for triple patterning lithography," in [Proc. DAC], 1181-1186 (2012).

[10] Ma, Q., Zhang, H., and Wong, M., "Triple patterning aware routing and its comparison with double patterning aware routing in $14 \mathrm{~nm}$ technology," in [Proc. DAC], 591-596 (2012).

[11] Tian, H., Zhang, H., Ma, Q., Xiao, Z., and Wong, M., "A polynomial time triple patterning algorithm for cell based row-structure layout," in [Proc. ICCAD], 57-64 (2012).

[12] Lin, Y.-H., Yu, B., Pan, D. Z., and Li, Y.-L., "TRIAD: a triple patterning lithography aware detailed router," in [Proc. ICCAD], 123-129 (2012).

[13] Kuang, J. and Young, E., "An efficient layout decomposition approach for triple patterning lithography," in [Proc. $D A C], 69: 1-69: 6(2013)$.

[14] Zhang, Y., Luk, W.-S., Zhou, H., Yan, C., and Zeng, X., "Layout decomposition with pairwise coloring for multiple patterning lithography," in [Proc. ICCAD], 170-177 (2013).

[15] Yu, B., Lin, Y.-H., Luk-Pat, G., Ding, D., Lucas, K., and Pan, D. Z., "A high-performance triple patterning layout decomposer with balanced density," in [Proc. ICCAD], 163-169 (2013).

[16] Tian, H., Du, Y., Zhang, H., Xiao, Z., and Wong, M. D., "Constrained pattern assignment for standard cell based triple patterning lithography," in [Proc. ICCAD], 178-185 (2013). 
[17] Yu, B., Xu, X., Gao, J.-R., and Pan, D. Z., "Methodology for standard cell compliance and detailed placement for triple patterning lithography," in [Proc. ICCAD], 349-356 (2013).

[18] Matsui, T., Kohira, Y., Kodama, C., and Takahashi, A., "Positive semidefinite relaxation and approximation algorithm for triple patterning lithography," in [Proc. ISAAC, LNCS 8889], 365-375 (2014).

[19] Yu, B., Gao, J.-R., and Pan, D. Z., "Triple patterning lithography (TPL) layout decomposition using end-cutting," in $[$ Proc. SPIE $], \mathbf{8 6 8 4}, \mathbf{8 6 8 4 0 G}(2013)$.

[20] Kohira, Y., Matsui, T., Yokoyama, Y., Kodama, C., Takahashi, A., and Shigeki Nojima, S. T., "Fast mask assignment using positive semidefinite relaxation in lelecut triple patterning," in [Proc. ASPDAC], 665-670 (2015).

[21] Kodama, C., Ichikawa, H., Nakayama, K., Kotani, T., Nojima, S., Mimotogi, S., Miyamoto, S., and Takahashi, A., "Self-aligned double and quadruple patterning aware grid routing with hotspots control," in [Proc. ASP-DAC], 267-272 (2013).

[22] Yu, B., Gao, J.-R., Ding, D., Ban, Y., Yang, J.-S., Yuan, K., Cho, M., and Pan, D. Z., "Dealing with ic manufacturability in extreme scaling," in [Proc. ICCAD], 240-242 (2012).

[23] Takahashi, A., Awad, A., Kohira, Y., Matsui, T., Kodama, C., Nojima, S., and Tanaka, S., "Multi patterning techniques for manufacturability enhancement in optical lithography," in [Proc. ICDV], 117-122 (2014).

[24] Goemans, M. X. and Williamson, D. P., "Improved approximation algorithms for maximum cut and satisfiability problems using semidefinite programming," Journal of the ACM 42, 1115-1145 (1995).

[25] "SDPA 7.3.8." http://sdpa.sourceforge.net/.

[26] "CPLEX 12.6.1." http://www-01.ibm.com/software/commerce/optimization/cplex-optimizer/. 